\title{
A case of intracranial molluscum contagiosum virus infection diagnosed by metagenomic sequencing of cerebrospinal fluid
}

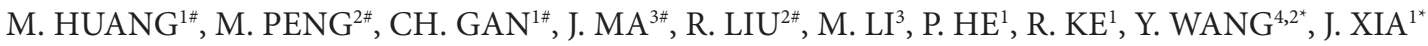

${ }^{1}$ Department of Infectious Diseases, The Fifth Affiliated Hospital, Sun Yat-Sen University, Zhuhai, P. R. China, 519000; ${ }^{2}$ Fifth Affiliated Hospital-BGI Laboratory, Center for Interventional Medicine, The Fifth Affiliated Hospital, Sun Yat-Sen University, Zhuhai, P. R.

China; ${ }^{3}$ BGI-Shenzhen, Shenzhen, P. R. China; ${ }^{4}$ Xinhua College, Sun Yat-sen University, Guangzhou, P. R. China

\begin{abstract}
Summary. - Molluscum contagiosum is a common, self-limiting infectious disease of the skin caused by molluscum contagiosum virus (MCV). The disease primarily affects children, sexually active adults, and immunocompromised individuals. Transmission of the virus occurs by direct skin contact. Therefore, the virus is usually detected in the skin and genitals of patients. However, the diagnosis of intracranial infection by the virus is difficult if the skin/mucosa lessons are atypical or absent, and the presence of the virus in the cerebrospinal fluid has not been reported. We report a very rare case of intracranial infection by molluscum contagiosum virus. A 25-year-old girl was admitted to our hospital due to severe headache but no fever or other symptoms. Upon examination, some small flesh-colored flattened papules on both arms were noticed. Blood tests showed slightly reduced levels of CD3 and CD4 T lymphocytes. Three-dimensional time-of-flight magnetic resonance angiography (3D-TOF-MRA) and head magnetic resonance (MR) were both normal. Lumbar puncture was performed, and metagenomic sequencing was applied to the spinal fluid. The unique sequences of the molluscum contagiosum virus were identified in the fluid. The patient was then diagnosed with intracranial molluscum contagiosum virus infection. No special treatment was given. The headache gradually disappeared, and the patient was discharged. During our quarterly follow-up, the girl appeared normal, and her skin lesions disappeared. However, her CD3 and CD4 T lymphocyte counts were still slightly lower than the normal level. Our case shows that the application of metagenomic sequencing to cerebrospinal fluid is a sensitive and powerful means to detect pathogens causing intracranial infection.
\end{abstract}

Keywords: Molluscum contagiosum; intracranial infection; metagenomics sequencing

Molluscum contagiosum is a benign viral papular eruption characterized by small, discrete, waxy, flesh-colored papules with central umbilication (Chen et al., 2013). The disease primarily affects school-going children (grade 1-5) and sexually active or immunocompromised adults (Chen et al., 2013). Lesions are frequently observed on the skin of the face, trunk and extremities in children or on the

*Corresponding author. E-mail: xiajinyu@mail.sysu.edu.cn (J. Xia), ywzhong@hotmail.com (Y. Wang); phone: +86-0756-2528570, +86-0756-2528712. "Mingxing Huang, Maochen Peng, Chongjie Gan, Jinmin Ma, and Ruihong Liu contributed equally to this work. Abbreviations: $\mathrm{MCV}=$ molluscum contagiosum virus; $\mathrm{NGS}=$ next generation sequencing genitals of young adults and rarely on the palms, soles and mucous membranes (Chen et al., 2013; Zhuang et al., 2015). Therefore, the virus that causes this disease is usually found on the skin and mucosa. Intracranial infection by the virus is very rare, and the detection of the virus in cerebrospinal fluid has not been previously reported. We present a case of intracranial molluscum contagiosum infection, comprising the symptoms, diagnosis, treatment and outcome.

A 25-year-old girl presented to our outpatient department due to severe headache. The headache had started 7 days prior and gradually intensified. She presented with no fever, vomiting, cough, unconsciousness or any psychoneural symptoms. On examination, several small, flesh-colored, flattened papules on both arms were found; these papules 
Table 1. The counts of T lymphocyte subsets were less than the lower limits of normal in the patient, while the cerebrospinal fluid appeared normal

\begin{tabular}{|c|c|c|c|}
\hline Items & Result & & $\begin{array}{c}\text { Normal } \\
\text { range }\end{array}$ \\
\hline \multicolumn{4}{|l|}{ T lymphocyte subsets } \\
\hline Absolute count of CD3+ cells & 745 & cells $/ \mu \mathrm{l}$ & $955-2600$ \\
\hline CD3+T cells/lymphocytes & $70.27 \%$ & & $50-84$ \\
\hline Absolute count of CD $3+\mathrm{CD} 4+$ cells & 337 & cells $/ \mu \mathrm{l}$ & $550-1440$ \\
\hline CD3+CD4+T cells/lymphocytes & 31.83 & & $27-51$ \\
\hline Absolute count of CD3+CD8+ cells & 357 & cells $/ \mu \mathrm{l}$ & $320-1250$ \\
\hline CD3+CD8+T cells/lymphocytes & $33.73 \%$ & & $15-41$ \\
\hline $\mathrm{CD} 3+\mathrm{CD} 4+/ \mathrm{CD} 3+\mathrm{CD} 8+$ & 0.94 & & $0.71-2.78$ \\
\hline \multicolumn{4}{|l|}{ Cerebrospinal fluid } \\
\hline Color & colorless & & \\
\hline Clarity & hyaline & & \\
\hline Clot & no & & \\
\hline $\mathrm{pH}$ value & 8.0 & & \\
\hline Proportion & 1.020 & & \\
\hline Pandy test & positive & & \\
\hline Erythrocyte microscopic examination & $0 / \mathrm{HP}$ & & \\
\hline Leucocyte microscopic examination & 0/HP & & \\
\hline Chloridion & 123.00 & $\mathrm{mmol} / \mathrm{l}$ & \\
\hline Glucose & 3.80 & $\mathrm{mmol} / \mathrm{l}$ & \\
\hline $\mathrm{LDH}$ & 11.00 & $\mathrm{U} / \mathrm{l}$ & \\
\hline Albumin & 0.415 & $\mathrm{~g} / \mathrm{l}$ & \\
\hline
\end{tabular}

appeared at approximately the same time as her headache. The lesions were not pruritic or painful. The patient was a virgin and denied recent trauma or skin contact with persons who had similar skin lesions.

The patient was then admitted to our Infectious Diseases Department. Three-dimensional time-of-flight magnetic resonance angiography (3D-TOF-MRA) and head magnetic resonance (MR) imaging were performed. The results of both examinations were normal. However, blood tests showed reduced CD3 and CD4 T lymphocyte counts (Table 1). We therefore performed a lumbar puncture. The physical and biochemical analyses showed a positive Pandy test (i.e. proteins were present in the cerebrospinal fluid) and otherwise normal results (Table 1 )

In addition to the physical and biochemical examinations, metagenomic sequencing was applied to the cerebrospinal fluid to detect the presence of pathogens. DNA was extracted from the fluid using the QIAamp DNA Mini Kit (Qiagen) according to the manufacturer's instructions, and next-generation sequencing (NGS) was performed on the BGISEQ-50 platform. Low-quality reads and reads from human hosts were excluded for further analysis. Finally, the remaining reads were classified by simultaneous alignment to a microbial genome database (in house) consisting of (a)

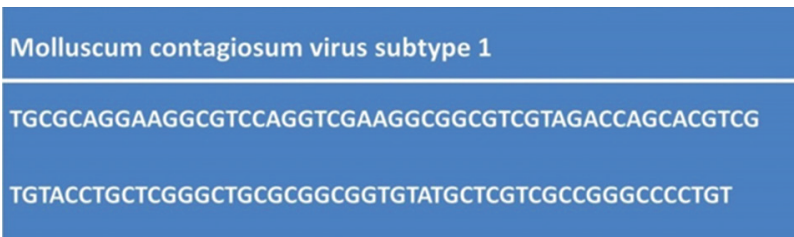

(b)
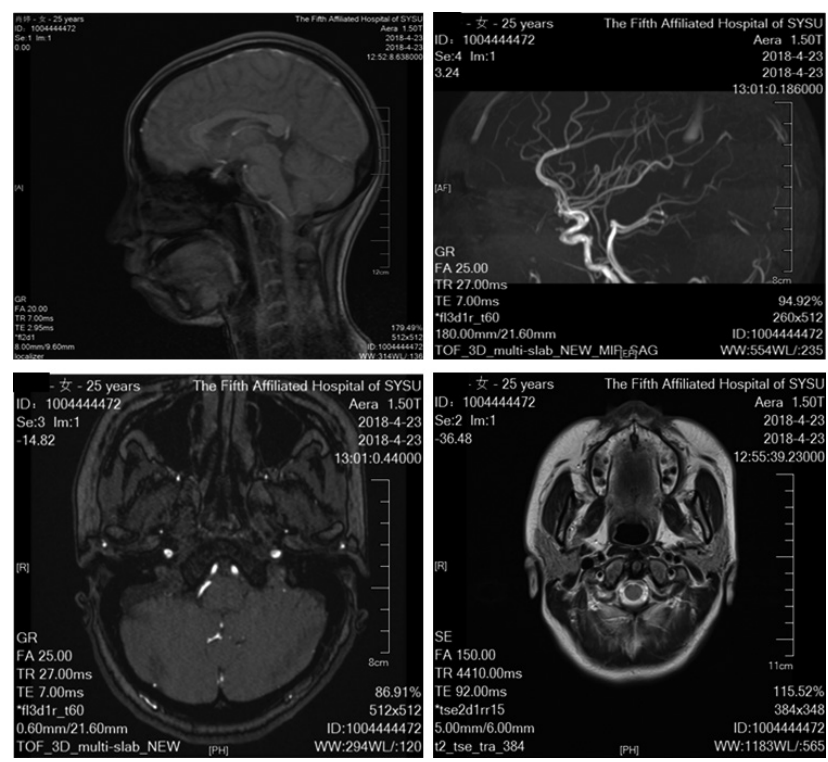

Fig. 1

(a) The gene sequence of MCV in the cerebrospinal fluid. (b) Threedimensional time-of-flight magnetic resonance angiography (3DTOF-MRA) and head magnetic resonance (MR) were performed on the patient and were both normal.

viruses, bacteria, fungi, and parasites. Two sequence reads were uniquely mapped to the molluscum contagiosum virus genome (Fig. 1a).

We then diagnosed the patient with an intracranial molluscum contagiosum virus infection. No special treatment was prescribed, but treatments to alleviate the symptoms were given. The headache disappeared 10 days after admission, and the skin lesions recovered 15 days after admission. The patient was then discharged from the hospital. Our quarterly follow-up showed that the girl remained healthy, except that her CD3 and CD4 T lymphocytes were still slightly lower than the normal level.

Molluscum contagiosum is a common benign infectious skin disease caused by the molluscum contagiosum virus, a member of the linear double-stranded DNA poxvirus family (Chen et al., 2013). The skin lesions are characterized by small, discrete, waxy, flesh-colored papules, an average of $3-5 \mathrm{~mm}$ in diameter and are usually solitary or in clusters 
(Bandino et al., 2011). When the lesions are squeezed or traumatized, a white curdy-type material can be extruded (Chen et al., 2013). The skin lesions are usually asymptomatic, although a minority of patients have complained about tenderness or pruritus (Bandino et al., 2011). The most commonly affected skin sites are the trunk, arms, and face in children and the genitals in sexually active adults (Chen et al., 2013). The disease can develop at any age but predominantly affects three distinct patient populations: children, sexually active adults, and immunocompromised individuals. In immunocompetent individuals, the number of molluscum contagiosum lesions is generally less than 20 (Chen et al., 2013). However, immunocompromised patients with HIV infection or those with atopic dermatitis may develop widespread, persistent and large lesions of molluscum contagiosum (Ianhez et al., 2011). Our patient was sexually inactive but had reduced CD3 and CD4 lymphocytes, indicating that she was immunocompromised, although she had no symptoms or signs suggestive of any other infection. The virus is transmitted by direct contact with an infected person or contaminated objects; evidence also indicates occasional transmission via fomites such as bath sponges and towels. Our patient could not recall having contact with any infected people, which is consistent with the usual difficulty in tracing the origins of the infection in immunocompromised individuals.

The clinical diagnosis of molluscum contagiosum is based on typical skin lesions and umbilicus papules. Histological and microbiome examinations are often unnecessary (Hoy et al., 2013). The differential diagnosis considers extensive papules and benign adnexal tumor, condyloma accuminatum, chickenpox (infection with varicella zoster virus), plane warts, cutaneous fungal infections, keratoacanthoma, leiomyoma, verruca vulgaris, papilloma, papular granuloma annulare, Paget's disease and other possibilities (Bandino et al., 2011; Hanson and Diven, 2003). The skin lesions in our patient were local, mild and atypical, and her predominant complaint was severe headache. However, she had no signs or symptoms of other diseases that may have caused the clinical picture. Metagenomic sequencing of her cerebrospinal fluid detected unique sequences of the virus. Together with the skin lesions and the exclusion of the other differential diagnoses, the metagenomic result enabled us to diagnose the patient with intracranial molluscum contagiosum virus infection. These findings show the power of metagenomic sequencing in detecting pathogens that cause intracranial infections. To the best of our knowledge, this is the first report of the molluscum contagiosum virus identified in cerebrospinal fluid. As metagenomic sequencing has not been used in most cerebrospinal fluid examinations and because molluscum contagiosum is self-limiting, the rate of infection in the central nervous system may be high. Metagenomic sequencing is a reliable method for detecting this virus and other pathogens, such as amoeba protozoa (Wang et al., 2018), in the cerebrospinal fluid.

The medical treatment of molluscum contagiosum is somewhat controversial. The lesions are benign and usually self-limiting. The average duration of a single lesion in immunosuppressed patients is approximately 2 months (Gottlieb and Myskowski, 1994; Chen et al., 2013); however, as the infection can be transmitted by autoinoculation via scratching or trauma, the mean duration of the infection is approximately 8 months, and several cases may persist for 12 months or longer (Gur, 2008). Our patient strictly followed the doctor's instructions after diagnosis. Her infection was quickly self-limited in 1 month. No evidence-based consensus has been reached on the best treatment for immunocompetent patients with molluscum contagiosum (Chen et al., 2013). In immunocompromised individuals with HIV infection, the presence of molluscum contagiosum lesions seems to indicate advancing immunosuppression (Valentine and Diven, 2000). For patients with HIV, the evidence suggests that systemic immune-modulating and antiviral drugs can be more effective than local ablative therapies. We did not give our patient any special treatment, but we did prescribe symptom-alleviating treatments, and the patient recovered, which further confirms our diagnosis. Our diagnostic approach, cerebrospinal fluid coupled with unbiased nextgeneration sequencing (NGS), provided a rapid and sensitive diagnosis of pathogens causing intracranial infection that eluded conventional testing for weeks after the initial presentation. NGS enables to identify both common and rare pathogens without any hypothesis. Especially in cerebrospinal fluid sample, some normal pathogens or non-pathogenic microbes were detected, like neurobrucellosis (Fan et al., 2018)2016 and June 1, 2017. The clinical characteristics and NGS results of patients with the diagnosis of NB were reviewed in this study. Results Four patients were rapidly diagnosed with NB using NGS of the CSF in patients with clinically suspected CNS infections, although the clinical manifestations varied dramatically between these patients. NGS of the CSF revealed that the sequence reads identified that corresponded to Brucella species ranged from 11 to 104 , with genomic coverage ranging from $0.043 \%$ to $0.4 \%$. Rapid diagnosis led to prompt treatment with the appropriate antibiotics. Conclusions This study demonstrates the power of NGS of the CSF coupled with a bioinformatic pipeline in the diagnosis of NB.",')author":[\{“dropping-particle":"',', fami ly":"Fan','given":"'Siyuan"',"non-dropping-particle":"’','parsenames":false,"suffix":"'”\},\{“dropping-particle":"’'”family":” Ren'"'given":"Haitao"',non-dropping-particle":"',"'parse-na mes":false,"suffix":"”\},\{“dropping-particle":"','family":"W ei",'given":"Yanping","non-dropping-particle":"',"parse-na mes":false,"suffix":"”\},\{“dropping-particle":"”','family":"M ao"',"given":"Chenhui"”,non-dropping-particle":"',"parsenames":false,"suffix":"”\},\{“dropping-particle":"'”',family": 
"Ma"'”given":"Zhenzi"',non-dropping-particle":"',"'parsenames":false,"suffix":"”’\},\{ddropping-particle”.'”'”family”:"Z hang,"'given":"Lu","non-dropping-particle":"',"'parse-names ":false,"suffix":"'\},\{“dropping-particle":"','family":"Wang","given":"Li","non-dropping-particle":"',"parse-nam es":false,"suffix":"”\},\{“dropping-particle":"'”,'family":"G e"'"given":"Ying"',non-dropping-particle":"’’"parse-nam es":false,"suffix":"'\},\{“dropping-particle":"','family":"L i","given":" Taisheng","non-dropping-particle":"',"'parsenames":false,"suffix":"”\},\{“dropping-particle":"’'”family":"C ui"',given":"Liying,"'non-dropping-particle":"',"parse-nam

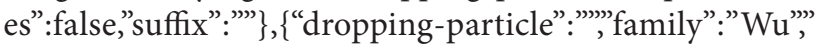
given":"Honglong,"”non-dropping-particle":"'”,"parse-nam es":false,"suffix":"”’\},\{“dropping-particle”:"'”'’family”:"Guan "','given":"Hongzhi"'”non-dropping-particle":"',"'parse-nam es":false,"suffix":"'’\}],"container-title":"International Journal of Infectious Diseases"',id":"ITEM-1'”'issued": ‘“dateparts":[[“2018"]]\},"title":"Next-generation sequencing of the cerebrospinal fluid in the diagnosis of neurobrucellos is"'”type":"article-journal"\},"uris":["http://www.mendeley. com/documents/?uuid=1772b866-febd-43a5-8cd4-fb447 ae6032b"]\}],"mendeley":\{“formattedCitation":"(Fan et al. 2018 or Propionibacterium acnes (Ye et al., 2016)current diagnostic methods are of limitation in many aspects, such as detecting range, time-consuming, specificity and sensitivity. In this report, we apply our new-developing pathogen detection method to clarify that Propionibacterium acnes is the causative agent of a two-year-old boy with juvenile myelomonocytic leukemia presenting clinical symptoms including serious rash and hyperpyrexia while traditional clinical methods of diagnosis fail to detect the pathogenic agent and multiple antimicrobial drugs are almost ineffective Propionibacterium acnes is confirmed to be the infectious agent by quantitative real-time polymerase chain reaction. $\mid \backslash n \backslash \backslash n C A S E$ PRESENTATION: After haploidentical hematopoietic stem cell transplantation, a two-year-old boy with juvenile myelomonocytic leukemia presented to a pediatrist in a medical facility with hyperpyrexia and red skin rash which later changed to black skin rash all over his body. Traditional diagnostic assays were unrevealing, and several routine antimicrobial treatments were ineffective, including the vancomycin, meropenem, tobramycin, cefepime and rifampin. In this case, pediatrist resorted to the nextgeneration sequencing technology for uncovering potential pathogens so as to direct their use of specific drugs against pathogenic bacteria. Therefore, based on the BGISEQ100 (Ion Proton System. These were supposed to be abnormal in cerebrospinal fluid in the past, but more and more cases were reported by metagenomics sequencing. What's more, the pseudorabies virus was detected in endophthalmitis (Ai et al., 2018) by NGS. That is unbelievable if there was no prompt NGS. In our case, molluscum contagiosum is also a normal pathogen but it is rare to detect the infection in the cerebrospinal fluid. Besides, the NGS results can provide information about abundance of pathogen, it can be viewed like a semi-quantitive sequencing platform that dynamically monitors the disease progression (Ai et al., 2017)"type":"article-newspaper"\},"uris":["http://www. mendeley.com/documents/?uuid=3fdc8cbd-8105-3f969c57-280c18abe938"]\}],"mendeley":\{“formattedCitation": "(Ai et al. 2017. Because the unbiased characteristic, even if the NGS is negative, it also might help to identify a noninfectious disease like tumor.

\section{Conclusion}

We report a very rare case of intracranial molluscum contagiosum virus infection. The patient presented with headache, atypical skin lesions of molluscum contagiosum and reduced CD 3 and CD 4 cell counts. Metagenomic sequencing applied to the cerebrospinal fluid detected sequences unique to the molluscum contagiosum virus. We diagnosed the patient with an intracranial molluscum contagiosum virus infection. The patient recovered with no special treatment, and this finding confirmed our diagnosis. Our experience showed the usefulness and power of the application of metagenomic sequencing to cerebrospinal fluid for detecting pathogens that cause intracranial infections.

Acknowledgments. This work was supported by grants from the National Key R\&D Program of China (No. 2016YFC1200105) and Science and Technology Innovation Foundation of Shenzhen, China (No. JCYJ20170817145915789). The funding agents had no role in study design, data collection and analysis, data interpretation, decision to publish, or preparation of the manuscript. We thank the patient who provided permission to show the results of the image and laboratory tests. We thank the National Key R\&D Program of China (No. 2016YFC1200105) and Science and Technology Innovation Foundation of Shenzhen, China (No. JCYJ20170817145915789) for their support.

\section{References}

Ai JW, Zhang HC, Cui P, Xu B, Gao Y, Cheng Q, Li T, Wu H, Zhang WH (2018) Dynamic and direct pathogen load surveillance to monitor disease progression and therapeutic efficacy in central nervous system infection using a novel semi-quantitive sequencing platform. J. Infect. 76, 307-310. https://doi.org/10.1016/j.jinf.2017.11.002

Ai JW, Weng SS, Cheng Q, Cui P, Li YJ, Wu HL, Zhu YM, Xu B, Zhang WH(2018) Human Endophthalmitis Caused By Pseudorabies Virus Infection, China, 2017. Emerg. Infect. Dis. 24, 1087-1090. https://doi.org/10.3201/ eid2406.171612 
Bandino JP, Wohltmann WE, Hivnor CM (2011) What is your diagnosis? Giant molluscum contagiosum. Cutis 88 , $164,170-172$

Chen X, Anstey AV, Bugert JJ (2013) Molluscum contagiosum virus infection. Lancet Infect. Dis., 13, 877-888. https:// doi.org/10.1016/S1473-3099(13)70109-9

Fan S, Ren H, Wei Y, Mao C, Ma Z, Zhang L, Wang L, Ge Y, Li T, Cui L, Wu H, Guan H (2018) Next-generation sequencing of the cerebrospinal fluid in the diagnosis of neurobrucellosis. Int. J. Infect. Dis. 67, 20-24. https://doi.org/10.1016/j. ijid.2017.11.028

Gottlieb SL, Myskowski PL (1994) Molluscum contagiosum. Int. J. Dermatol., 33, 453-461. https://doi. org/10.1111/j.1365-4362.1994.tb02853.x

Gur I (2008) The epidemiology of Molluscum contagiosum in HIV-seropositive patients: a unique entity or insignificant finding? Int. J. STD AIDS 19, 503-506. https://doi. org/10.1258/ijsa.2008.008186

Hanson D, Diven DG (2003) Molluscum contagiosum. Dermatol. Online J., 9, 2.

Hoyt BS, Tschen JA, Cohen PR (2013) Molluscum contagiosum of the areola and nipple: case report and literature review. Dermatol. Online J. 19, 18965.
Ianhez M, Cestari SC, Enokihara MY, Seize MB (2011) Dermoscopic patterns of molluscum contagiosum: a study of 211 lesions confirmed by histopathology. An. Bras. Dermatol. 86, 74-79. https://doi.org/10.1590/S0365$\underline{05962011000100009}$

Valentine CL, Diven D (2000):. Treatment modalities for molluscum contagiosum. Dermatol. Ther. 13, 285-289. https:// doi.org/10.1046/j.1529-8019.2000.00030.x

Wang Q, Li J, Ji J, Yang L, Chen L, Zhou R, Yang Y, Zheng H, Yuan J, Li L, Bi Y, Gao GF, Ma J, Liu Y (2018) A case of Naegleria fowleri related primary amoebic meningoencephalitis in China diagnosed by next-generation sequencing. BMC Infect. Dis. 18, 349. https://doi.org/10.1186/s12879-018$\underline{3261-Z}$

Ye M, Wei W, Yang Z, Li Y, Cheng S, Wang K, Zhou T, Sun J, Liu S, Ni N, Jiang H, Jiang H (2016) Rapid diagnosis of Propionibacterium acnes infection in patient with hyperpyrexia after hematopoietic stem cell transplantation by next-generation sequencing: a case report. BMC Infect. Dis. 16, 5. https://doi.org/10.1186/s12879-015-1306-0

Zhuang K, Ran Y, Xu F, Lama J (2015) Atypical infantile genital Molluscum contagiosum. An. Bras. Dermatol. 90, 403-405. https://doi.org/10.1590/abd1806-4841.20153298 